\title{
Lipid metabolic pathways as lung cancer therapeutic targets: A computational study
}

\author{
KOJIRO YANO \\ Faculty of Information Science and Technology, Osaka Institute of Technology, \\ 1-79-1 Kitayama, Hirakata-City, Osaka, Japan
}

Received November 3, 2011; Accepted December 13, 2011

DOI: $10.3892 / \mathrm{ijmm} .2011 .876$

\begin{abstract}
Inhibitors of lipid metabolic pathways, particularly drugs targeting the mevalonate pathway, have been suggested to be valuable in enhancing the effectiveness of epidermal growth factor receptor-tyrosine kinase inhibitors (EGFR-TKIs) and these compounds may also be effective in patients with inherent or acquired resistance to EGFR-TKIs. The present study examined gene expression profiles in lung adenocarcinoma to characterize the interaction between growth factor signals and lipid metabolic pathways at the transcriptional level. Gene expression correlation analysis showed that genes involved in the mevalonate pathway and unsaturated fatty acid synthesis were negatively correlated with the expression of EGFR, MET and other growth factor receptor genes, as well as with the expression of genes involved in cell migration and adhesion. On the other hand, the expression of genes related to cell cycle progression, DNA repair and DNA replication were positively correlated with the metabolic pathway genes mentioned above, and a significant number of such genes had promoter domains for nuclear factor Y (NFY). Genes whose expression showed a positive correlation with NFY expression and mevalonate pathway genes were found to exhibit protein-protein interactions with several 'hub' genes, including BRCA1, that have been associated with both lung cancer and cell division. These results support the idea that inhibition of lipid metabolic pathways may be valuable as an alternative therapeutic option for the treatment of lung adenocarcinoma, and suggest that NFY is a possible molecular target for such efforts.
\end{abstract}

\section{Introduction}

Lung adenocarcinoma accounts for about half of all non-small cell lung cancer (NSCLC) cases and is one of the major causes of death in developed countries (1). Epidermal growth factor

Correspondence to: Dr Kojiro Yano, Faculty of Information Science and Technology, Osaka Institute of Technology, 1-79-1 Kitayama, Hirakata-City, Osaka, Japan

E-mail: kyano@is.oit.ac.jp

Key words: lung cancer, microarray, lipid metabolism receptor (EGFR) tyrosine kinase inhibitors (TKIs) have been intensively assessed over the past several years as targeted agents for advanced NSCLC. Whereas EGFR-TKIs are highly effective in the treatment of adenocarcinoma associated with specific EGFR mutations that cause sustained receptor activity, drug effectiveness is significantly lower in patients without the activating mutations, and even patients with the mutations frequently develop resistance to EGFR-TKI (2). Therefore, new therapeutic targets that can overcome inherent or acquired resistance to EGFR-TKIs are highly desirable. Recently, it has been suggested that acquired resistance to EGFR-TKIs may be related to amplification of a hepatocyte growth factor (HGF) receptor, termed MET (3). HGF expression can induce EGFR-TKI resistance to lung adenocarcinoma cells with EGFR-activating mutations (4), and MET inhibition can reduce proliferation of lung adenocarcinoma cell lines that show resistance to EGFR-TKIs (3). MET amplification occurs in about $20 \%$ of NSCLC patients and is associated with poor survival.

The lipid metabolism pathway may also modulate the effectiveness of EGFR-TKIs in lung adenocarcinoma patients. It has been suggested that lipid-lowering drug statins may reduce cancer risk (5), and a large case-control study of US veterans found that this may be true for lung cancer (6), although some reports claim otherwise $(7,8)$. In vitro studies have shown that inhibition of the mevalonate pathway by statins reduces EGFR autophosphorylation (9), downstream AKT signaling (10), and EGF-induced RhoA translocation to the plasma membrane (11). Enhancement of EGFR-TKI effectiveness by statins seems to occur not only in cells with EGFR-activating mutations but also in EGFR-TKI-resistant NSCLC cell lines (12). The mechanism of EGFR signaling inhibition is not fully characterized, but reduced prenylation of small GTP-binding proteins may be of importance (13). However, depletion of cholesterol in the plasma membrane is known to increase EGFR signaling activity, perhaps by releasing EGFR from lipid rafts and inhibiting receptor internalization $(14,15)$. This suggests that the lipid metabolism pathway can influence EGFR signaling in both a positive and negative manner.

This study sought to characterize the lipid metabolism pathway in lung adenocarcinoma using gene expression correlation analysis of microarray data. More specifically, pathway genes that show associations with EGFR or MET were examined in detail, because EGFR and MET are among 
the best-studied growth signals in lung cancer patients. Gene expression profiles have been used to classify lung cancer (16), to discover gene sets which are predictive of disease prognosis (17), and to investigate molecular mechanisms of disease progression (18). However, large-scale analysis of the association between metabolic and growth factor signaling pathways has not been conducted in lung cancer tissue. In the present study, a set of lipid metabolism pathway genes, the expression of which are highly correlated with EGFR or MET, were first selected. Next, genes in the microarray dataset showing significant correlation with selected genes were examined in terms of functional properties. Finally, possible regulatory mechanisms of correlated expression were inferred using known transcription factor target sequences. This type of analysis predicts how the lipid metabolic pathway may functionally interact with EGFR, MET, and other biological processes in lung cancer cells, and offers an insight into the roles of EGFR and MET inhibition in lung cancer therapeutics.

\section{Materials and methods}

Microarray data. The microarray dataset GSE10072 (19) from the Gene Expression Omnibus (20) was used for analysis. The dataset contains expression profiles of 58 tumor and 49 non-tumor tissues. The information was originally obtained using the Affymetrix Human Genome U133A Array. The data from 22,215 probes in the array were normalized using the quantile normalization function (quantilenorm) of the Matlab Bioinformatics Toolbox (MathWorks, Natick, MA).

Classification of genes by Gene Ontology. The DAVID functional annotation tool [version $6.7 \mathrm{~b}(21,22)]$ was used to classify gene sets by Gene Ontology identifiers or using UCSC transcription factor binding sites (23). Functional categories with a Benjamini-Hochberg statistic (24) of $<0.025$ were considered statistically significant.

Statistical analysis. Pearson correlation coefficients were calculated using the 'corr' function from Matlab. The 2.5th and 97.5th percentiles of coefficients for 100,000 pairwise combinations between randomly selected genes in the dataset were -0.379 and 0.428 , respectively, and these were used as threshold values for significantly negative and positive correlations. Two-sample t-testing was achieved using the 'ttest2' function from Matlab.

\section{Results}

Correlation of lipid metabolism genes with EGFR expression. A total of 301 genes classified as 'lipid metabolic process' (GO:0006629) by gene ontology were selected and Pearson correlation coefficients were calculated between the expression of such genes and EGFR and MET. Although no gene showed a positive correlation with EGFR or MET expression, eight and nine such genes displayed a negative correlation with EGFR and MET expression, respectively, in cancer samples (Table I). The negative correlations were not evident in normal lung samples, except for MVK, which showed a significant negative correlation with MET in both cancerous and normal cells. Among the negatively correlated genes, HMG-coenzyme

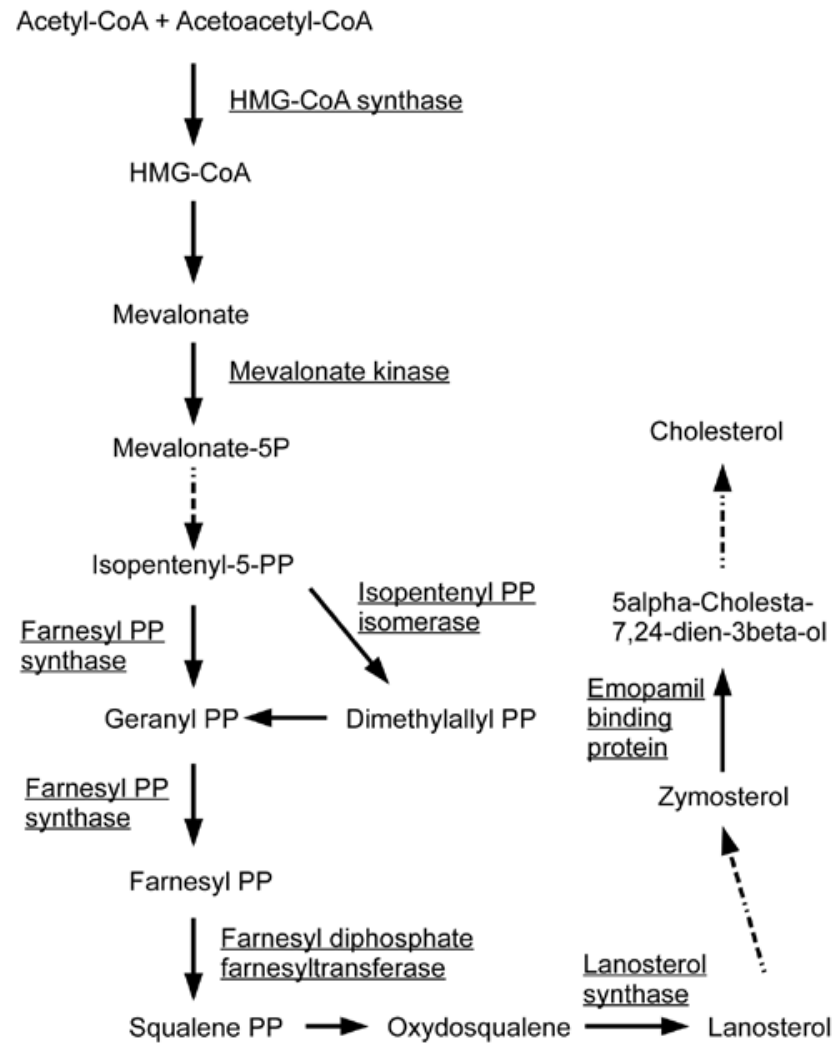

Figure 1. The mevalonate pathway. Enzymes, the encoding genes of which were negatively correlated with EGFR or MET expression are underlined. Broken arrows indicate that more than one reaction is involved. $\mathrm{P}$ and $\mathrm{PP}$ indicate phosphate and pyrophosphate, respectively.

A synthase 1 (HMGCS1), farnesyl-diphosphate farnesyltransferase 1 (FDFT1), farnesyl diphosphate synthase (FDPS), isopentenyl-diphosphate $\delta$ isomerase 1 (IDI1), lanosterol synthase (LSS), emopamil binding protein (EBP) and mevalonate kinase (MVK) are known to be involved in the first steps of steroid biosynthesis (Fig. 1). FAS and stearoyl-CoA desaturase (SCD) mediate the synthesis of monounsaturated fatty acids from acetyl-CoA, and fatty acid desaturase 1 (FADS1), fatty acid desaturase 2 (FADS2), and elongation of very long chain fatty acids (fen1/elo2, sur4/elo3, yeast)-like 2 (ELOVL2) catalyze the production of polyunsaturated fatty acids, including arachidonic acid (Fig. 2). Fatty acid 2-hydroxylase (FA2H) is involved in sphingolipid metabolism and mutations in this gene are known to cause leukodystrophy, whereas phosphatidylglycerophosphate synthase 1 (PGS1) is involved in glycerophospholipid metabolism, synthesizing phosphatidyl-glycerophosphate from CDP-diacylglycerol. These results suggest that EGFR and MET are closely, but negatively, associated with the expression of a variety of fatty acid biosynthesis genes in lung adenocarcinoma tissue.

Functional gene categories associated with lipid metabolism genes anti-correlated to EGFR. Next, associations of the 'anti-EGFR/MET' lipid metabolism genes with other genes were evaluated by calculation of the intergene Pearson correlation coefficients in lung cancer samples. Table II shows the number of genes demonstrating significant positive or negative associations with mevalonate pathway genes (FDFT1, FDPS, HMGCS1, IDI1, LSS, EBP and MVK). 
Table I. Lipid metabolic process genes negatively correlated with EGFR or MET.

\begin{tabular}{|c|c|c|c|}
\hline Symbol & Name & Cancer $^{\mathrm{a}}$ & Normal $^{\mathrm{a}}$ \\
\hline \multicolumn{4}{|l|}{ EGFR } \\
\hline FA2H & Fatty acid 2-hydroxylase & -0.442 & -0.073 \\
\hline FADS2 & Fatty acid desaturase 2 & -0.419 & 0.181 \\
\hline FDFT1 & Farnesyl-diphosphate farnesyltransferase 1 & -0.414 & -0.093 \\
\hline FDPS & Farnesyl diphosphate synthase & -0.402 & -0.321 \\
\hline HMGCS1 & HMG-coenzyme A synthase 1 & -0.559 & -0.224 \\
\hline IDI1 & Isopentenyl-diphosphate $\delta$ isomerase 1 & -0.438 & -0.084 \\
\hline LSS & Lanosterol synthase & -0.402 & -0.115 \\
\hline SCD & Stearoyl-CoA desaturase & -0.399 & -0.021 \\
\hline \multicolumn{4}{|l|}{ MET } \\
\hline $\mathrm{EBP}$ & Emopamil binding protein & -0.441 & 0.245 \\
\hline ELOVL2 & $\begin{array}{l}\text { Elongation of very long chain fatty acids } \\
\text { (fen1/elo2, sur4/elo3, yeast)-like } 2\end{array}$ & -0.427 & -0.146 \\
\hline FADS 1 & Fatty acid desaturase 1 & -0.411 & 0.115 \\
\hline FADS2 & Fatty acid desaturase 2 & -0.596 & -0.099 \\
\hline FASN & Fatty acid synthase & -0.526 & 0.108 \\
\hline IDI1 & Isopentenyl-diphosphate $\delta$ isomerase 1 & -0.463 & 0.301 \\
\hline LSS & Lanosterol synthase & -0.390 & 0.117 \\
\hline MVK & Mevalonate kinase & -0.380 & -0.391 \\
\hline PGS1 & Phosphatidylglycerophosphate synthase 1 & -0.421 & -0.061 \\
\hline
\end{tabular}

${ }^{a}$ Values in the columns show the Pearson correlation coefficients for the respective growth factor genes.

A

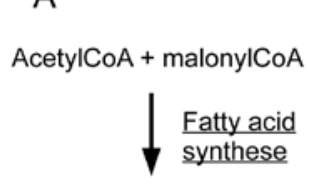

Palmitic acid

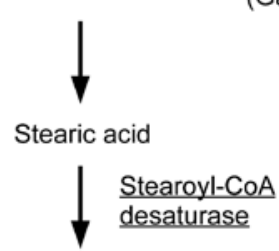

Oleic acid
B

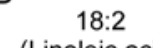

(Linoleic acid)

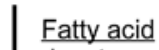

desaturase 2

$18: 3$

(Gamma-linoleic acid)

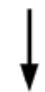

20:3

Fatty acid

desaturase

20:4

(Arachidonic acid)

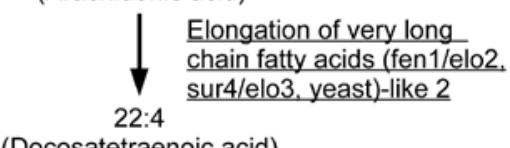

(Docosatetraenoic acid)

Figure 2. Fatty acid synthesis pathways. Enzymes, the encoding genes of which were negatively correlated with EGFR or MET expression are underlined. (A) Synthesis of a monounsaturated fatty acid, using oleic acid as an example. (B) Synthesis of a polyunsaturated fatty acid, using docosatetraenoic acid as an example.

Among these seven genes, FDPS, HMGCS1, IDI1 and MVK, all of which mediate farnesyl pyrophosphate synthesis from mevalonate, showed particularly large numbers of correlated genes. In addition, 166 genes in the microarray dataset displayed significant positive associations with three or more of the mevalonate pathway genes. According to
Table II. Number of genes significantly correlated with lipid metabolism genes.

\begin{tabular}{lrr}
\hline Symbol & Positive & Negative \\
\hline Mevalonate pathway genes & & \\
FDFT1 & 68 & 162 \\
FDPS & 438 & 480 \\
HMGCS1 & 372 & 381 \\
IDI1 & 505 & 573 \\
LSS & 166 & 192 \\
EBP & 294 & 431 \\
MVK & 3012 & 1462 \\
Fatty acid synthesis pathways genes & & \\
ELOVL2 & 1428 & 459 \\
FA2H & 87 & 93 \\
FADS1 & 182 & 214 \\
FADS2 & 130 & 200 \\
FASN & 67 & 325 \\
PGS1 & 147 & 127 \\
SCD & 152 & 188 \\
\hline
\end{tabular}

DAVID, gene functional categories were dominated by GO Biological Processes related to the cell cycle, DNA replication, response to DNA damage, and lipid metabolism, suggesting close links between the regulation of cell division and cholesterol biosynthesis (Table III). On the other hand, 235 genes had significant negative associations with three or more of 
Table III. Enriched GO biological process terms showing significant correlations with mevalonate and fatty acid synthesis pathway genes.

\begin{tabular}{lc} 
Term & Count \\
\hline Enriched GO biological process terms in genes positively correlated with the mevalonate pathway genes \\
GO:0007049, cell cycle
\end{tabular}

67

GO:0000278, mitotic cell cycle

GO:0022403, cell cycle phase

GO:0007067, mitosis

GO:0000279, M phase

GO:0000087, M phase of mitotic cell cycle

GO:0022402, cell cycle process

GO:0051301, cell division

GO:0006259, DNA metabolic process

GO:0006260, DNA replication

GO:0000075, cell cycle checkpoint

GO:0007051, spindle organization and biogenesis

GO:0007017, microtubule-based process

GO:0000074, regulation of progression through cell cycle

GO:0051726, regulation of cell cycle

GO:0006996, organelle organization and biogenesis

GO:0000070, mitotic sister chromatid segregation

GO:0000819, sister chromatid segregation

GO:0007059, chromosome segregation

GO:0006974, response to DNA damage stimulus

GO:0000226, microtubule cytoskeleton organization and biogenesis

GO:0007088, regulation of mitosis

GO:0051276, chromosome organization and biogenesis

GO:0009719, response to endogenous stimulus

GO:0007093, mitotic cell cycle checkpoint

GO:0016126, sterol biosynthetic process

GO:0007010, cytoskeleton organization and biogenesis

GO:0051325, interphase

GO:0006281, DNA repair

GO:0006261, DNA-dependent DNA replication

GO:0016125, sterol metabolic process

GO:0051329, interphase of mitotic cell cycle

GO:0007052, mitotic spindle organization and biogenesis

GO:0006694, steroid biosynthetic process

GO:0006695, cholesterol biosynthetic process

GO:0006270, DNA replication initiation

GO:0009987, cellular process

GO:0008203, cholesterol metabolic process

GO:0044237, cellular metabolic process

GO:0016043, cellular component organization and biogenesis

GO:0006139, nucleobase, nucleoside, nucleotide and nucleic acid metabolic process

GO:0008202, steroid metabolic process

GO:0044238, primary metabolic process

GO:0048015, phosphoinositide-mediated signaling

GO:0006268, DNA unwinding during replication

GO:0031570, DNA integrity checkpoint

GO:0007018, microtubule-based movement

GO:0000910, cytokinesis

GO:0032508, DNA duplex unwinding

GO:0032392, DNA geometric change
46

45

38

41

38

55

37

43

24

15

11

22

31

31

44

11

11

13

23

14

13

24

23

9

9

24

12

17

12

11

11

6

10

7

7

150

9

112

52

68

12

111

9

5

6

9

6

5

5
$3.56 \mathrm{E}-37$

4.44E-36

1.01E-32

1.21E-31

1.34E-31

$1.42 \mathrm{E}-31$

2.26E-29

3.18E-29

1.37E-15

2.92E-13

$5.30 \mathrm{E}-13$

5.83E-13

2.24E-12

2.71E-12

2.92E-12

2.00E-11

8.75E-11

1.19E-10

$3.18 \mathrm{E}-10$

3.46E-10

4.49E-10

1.03E-09

2.02E-09

2.09E-08

2.01E-07

2.50E-07

5.16E-07

7.14E-07

2.01E-06

$2.29 \mathrm{E}-06$

2.68E-06

4.48E-06

1.13E-05

2.43E-05

2.70E-05

8.05E-05

$1.10 \mathrm{E}-04$

$1.25 \mathrm{E}-04$

3.23E-04

$3.59 \mathrm{E}-04$

3.63E-04

$3.69 \mathrm{E}-04$

7.69E-04

$1.33 \mathrm{E}-03$

$1.42 \mathrm{E}-03$

$1.75 \mathrm{E}-03$

$1.87 \mathrm{E}-03$

$1.96 \mathrm{E}-03$

2.19E-03

2.19E-03 
Table III. Continued.

\begin{tabular}{l} 
Term \\
\hline GO:0008610, lipid biosynthetic process \\
GO:0008152, metabolic process \\
GO:0008299, isoprenoid biosynthetic process \\
GO:0030705, cytoskeleton-dependent intracellular transport \\
GO:0031577, spindle checkpoint \\
GO:0006066, alcohol metabolic process \\
GO:0042770, DNA damage response, signal transduction \\
GO:0043283, biopolymer metabolic process \\
GO:0000077, DNA damage checkpoint \\
GO:0006950, response to stress \\
GO:0006720, isoprenoid metabolic process
\end{tabular}

$\begin{array}{rl}\text { Count } & \text { Benjamini } \\ 13 & 2.45 \mathrm{E}-03 \\ 117 & 3.33 \mathrm{E}-03 \\ 5 & 3.38 \mathrm{E}-03 \\ 9 & 5.04 \mathrm{E}-03 \\ 4 & 5.08 \mathrm{E}-03 \\ 13 & 9.20 \mathrm{E}-03 \\ 6 & 1.05 \mathrm{E}-02 \\ 77 & 1.33 \mathrm{E}-02 \\ 5 & 1.65 \mathrm{E}-02 \\ 25 & 1.67 \mathrm{E}-02 \\ 5 & 1.85 \mathrm{E}-02\end{array}$

Enriched GO biological process terms in genes negatively correlated with the mevalonate pathway genes

$\begin{array}{ll}\text { GO:0022610, biological adhesion } & 61\end{array}$

$1.51 \mathrm{E}-25$

GO:0007155, cell adhesion

$1.51 \mathrm{E}-25$

GO:0016337, cell-cell adhesion

$8.98 \mathrm{E}-17$

GO:0007156, homophilic cell adhesion

$4.41 \mathrm{E}-16$

GO:0009605, response to external stimulus

5.78E-06

GO:0009611, response to wounding

6.36E-06

GO:0048518, positive regulation of biological process

$3.50 \mathrm{E}-04$

GO:0032501, multicellular organismal process

$4.84 \mathrm{E}-04$

GO:0032502, developmental process

4.97E-04

GO:0006954, inflammatory response

$5.46 \mathrm{E}-04$

GO:0048731, system development

$5.76 \mathrm{E}-04$

GO:0048856, anatomical structure development

$6.46 \mathrm{E}-04$

GO:0048513, organ development

9.45E-04

GO:0006950, response to stress

$1.76 \mathrm{E}-03$

GO:0006952, defense response

$1.79 \mathrm{E}-03$

GO:0007275, multicellular organismal development

$1.80 \mathrm{E}-03$

GO:0008283, cell proliferation

$1.90 \mathrm{E}-03$

GO:0007167, enzyme linked receptor protein signaling pathway

$3.11 \mathrm{E}-03$

GO:0001944, vasculature development

4.01E-03

GO:0048522, positive regulation of cellular process

$4.99 \mathrm{E}-03$

GO:0048523, negative regulation of cellular process

9.29E-03

GO:0009887, organ morphogenesis

$1.50 \mathrm{E}-02$

GO:0001568, blood vessel development

$1.51 \mathrm{E}-02$

GO:0006959, humoral immune response

$1.52 \mathrm{E}-02$

GO:0048519, negative regulation of biological process

$1.77 \mathrm{E}-02$

Enriched GO biological process terms in genes positively correlated with fatty acid synthesis pathways genes

GO:0008610, lipid biosynthetic process

1.83E-03

GO:0000278, mitotic cell cycle

$2.01 \mathrm{E}-03$

GO:0007049, cell cycle

GO:0007051, spindle organization and biogenesis

2.03E-03

GO:0000226, microtubule cytoskeleton organization and biogenesis

$2.22 \mathrm{E}-03$

GO:0000087, M phase of mitotic cell cycle

$2.35 \mathrm{E}-03$

GO:0006695, cholesterol biosynthetic process

$2.47 \mathrm{E}-03$

GO:0007067, mitosis

$2.52 \mathrm{E}-03$

GO:0051301, cell division

$2.71 \mathrm{E}-03$

$3.06 \mathrm{E}-03$

GO:0016126, sterol biosynthetic process

$3.46 \mathrm{E}-03$

GO:0000279, M phase

$5.39 \mathrm{E}-03$

GO:0022403, cell cycle phase

$1.39 \mathrm{E}-02$

GO:0044255, cellular lipid metabolic process

$1.48 \mathrm{E}-02$ 
Table IV. Enriched GO biological process terms with the NFY-correlated genes.

\begin{tabular}{lcc}
\hline Term & Count & Benjamini \\
\hline GO:0006259, DNA metabolic process & 16 & $1.49 \mathrm{E}-04$ \\
GO:0006139, nucleobase, nucleoside, nucleotide & 31 & $4.00 \mathrm{E}-04$ \\
$\quad$ and nucleic acid metabolic process & 10 & $1.05 \mathrm{E}-03$ \\
GO:0006974, response to DNA damage stimulus & 40 & $1.12 \mathrm{E}-03$ \\
GO:0043170, macromolecule metabolic process & 9 & $1.24 \mathrm{E}-03$ \\
GO:0006260, DNA replication & 9 & $1.36 \mathrm{E}-03$ \\
GO:0006281, DNA repair & 10 & $2.74 \mathrm{E}-03$ \\
GO:0009719, response to endogenous stimulus & 41 & $1.07 \mathrm{E}-02$ \\
GO:0044238, primary metabolic process & 41 & $1.11 \mathrm{E}-02$ \\
GO:0044237, cellular metabolic process & 6 & $1.20 \mathrm{E}-02$ \\
GO:0006261, DNA-dependent DNA replication & 32 & $1.23 \mathrm{E}-02$ \\
GO:0043283, biopolymer metabolic process & 8 & $2.00 \mathrm{E}-02$ \\
GO:0016071, mRNA metabolic process & &
\end{tabular}

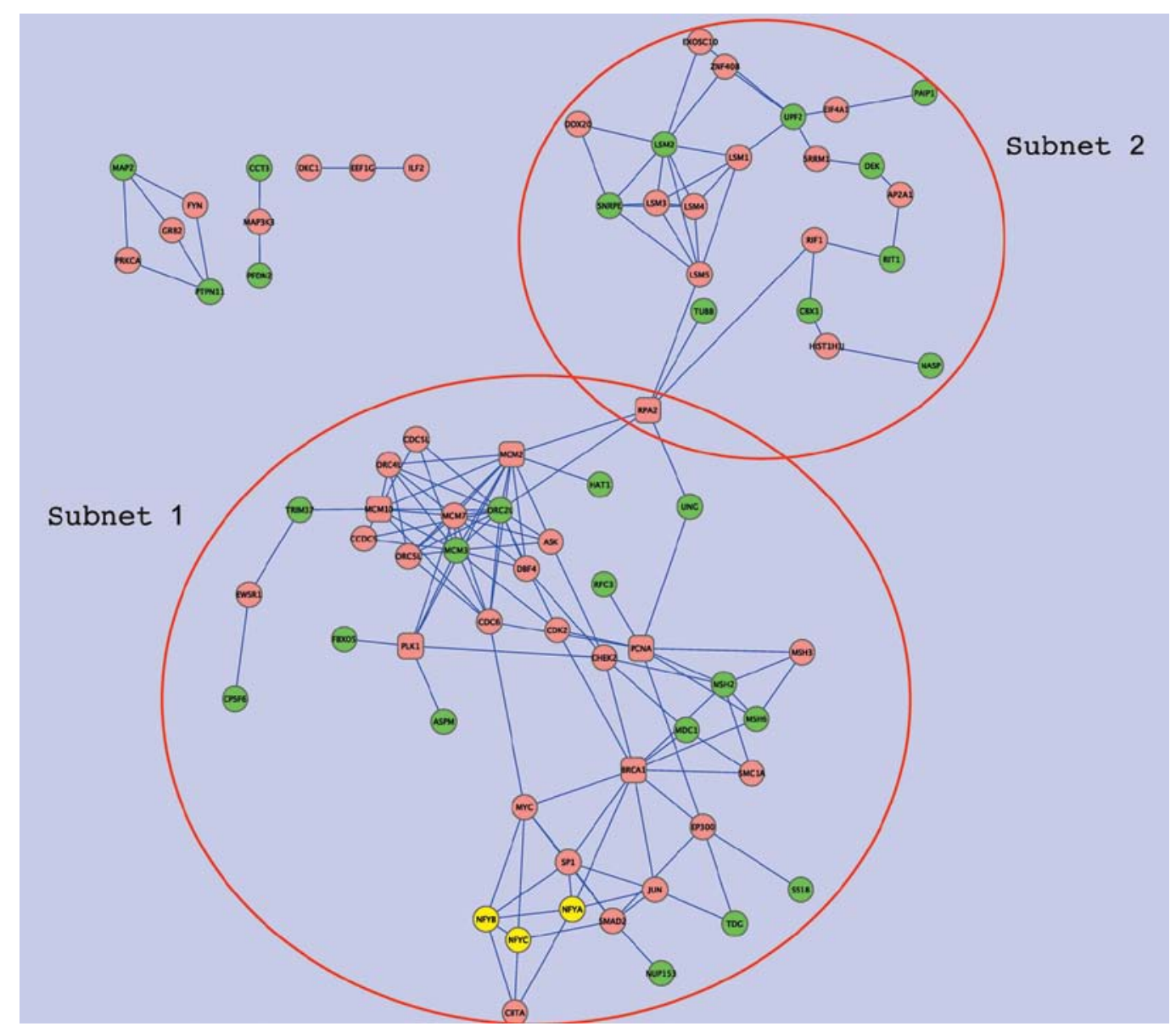

Figure 3. Protein-protein interaction network of NFY and associated genes. Yellow, green and pink nodes are NFY genes, genes correlated with NFY and mevalonate pathway genes, and genes with known protein-protein interactions with NFY genes or NFY-correlated genes. Red round rectangles are 'hub' genes (please see the main text). The red circles are subnets 1 and 2; these are shown on a larger scale in Figures 4 and 5, respectively.

the mevalonate pathway genes. The functional categories were principally related to cell adhesion, cell migration, blood vessel development, extracellular matrix synthesis, and defense responses (Table III). This gene set also included regulators of cell proliferation, including endothelin receptor type A (EDNRA), platelet-derived growth factor receptor, $\alpha$ polypeptide (PDGFRA), protein kinase $\mathrm{C} \alpha$ (PRKCA), rasrelated $\mathrm{C} 3$ botulinum toxin substrate 2 (RAC2), transforming growth factor $\beta$, receptor II (TGFBR2), and vitamin D receptor (VDR). These data may suggest that mevalonate pathway 


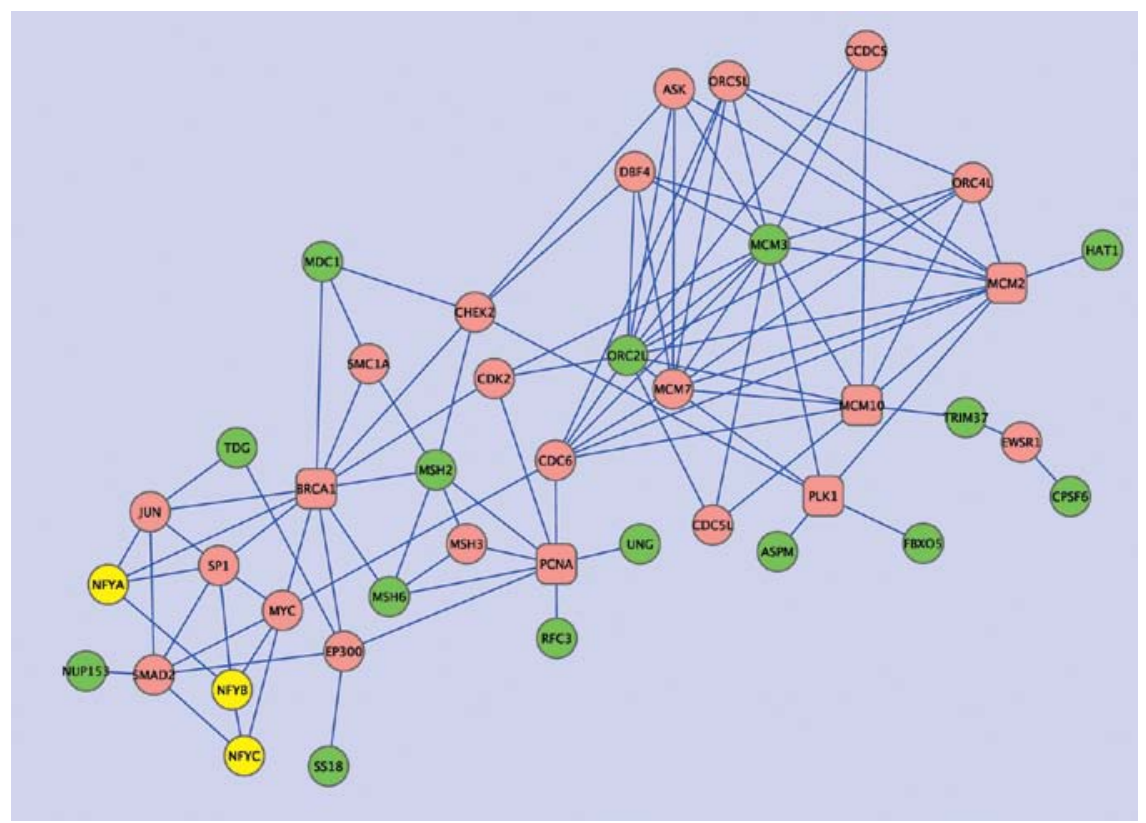

Figure 4. Subnet 1 of the protein-protein interaction network of NFY and associated genes. Notations for nodes are as in Figure 3.

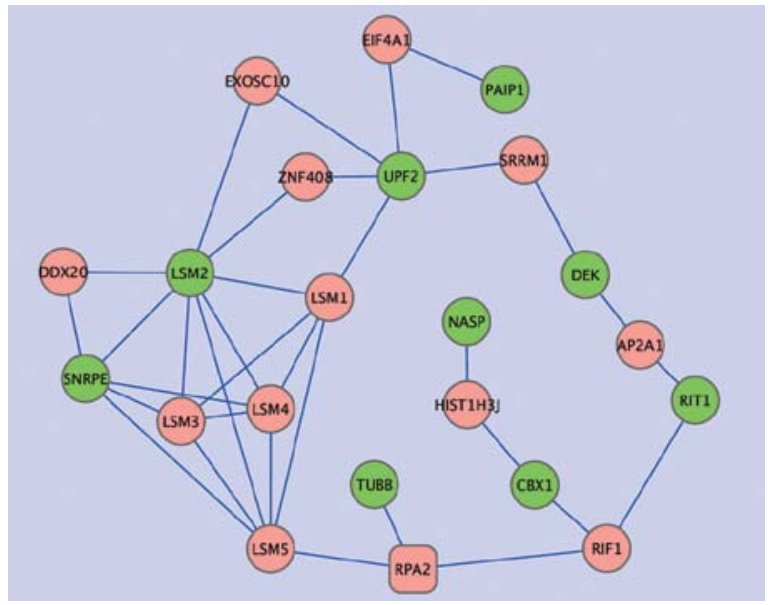

Figure 5. Subnet 2 of the protein-protein interaction network of NFY and associated genes. Notations for nodes are as in Figure 3.

genes were negatively associated with processes mediating signal transduction from the extracellular space, but positively associated with pathways involving the nucleus. Similarly, anti-EGFR/MET lipid metabolism genes involved in fatty acid synthesis (FADS1, FADS2, FASN, SCD, ELOVL2, PGS1 and FA2H) were evaluated (Table II). Most of these genes showed smaller numbers of correlations than genes of the mevalonate pathway. Only 18 and 35 genes displayed significant positive and negative correlations, respectively, with three or more of the fatty acid synthesis genes. The positively correlated genes belonged to sets of functional categories similar to those positively correlated with mevalonate pathway genes (Table III); these were genes of the cell cycle, cell division and lipid metabolism. No functional category was significantly enriched in negatively correlated genes.

Transcriptional regulatory mechanisms associated with antiEGFR lipid metabolism genes. Gene expression correlation analysis showed that lipid metabolism genes were associated with specific biological processes, particularly the cell cycle. To determine a possible mechanism of correlated expression, enrichment of predicted transcription factor binding sites was examined by DAVID. It was found that genes positively associated with mevalonate pathway genes were enriched in the NFY binding site, with a Benjamini score of 3.4E-8. To examine the relationship between NFY and genes positively correlated with mevalonate pathway genes, a search was instituted for genes showing significant positive correlations with NFY. As NFY is composed of subunits encoded by three genes, NFYA, NFYB and NFYC, genes with positive correlations with at least one subunit were selected. Respectively 202, 889 and 133 genes were found to display a correlation with NFYA, NFYB and NFYC, and, in total, 1,166 genes displayed significant positive correlations with one or more of the NYF subunit genes. For each gene identified, Pearson correlation coefficients were calculated with respect to genes positively correlated with mevalonate pathway genes, and the number of significant positive correlations was enumerated. This disclosed that 53 genes showed positive correlations with 81 or more of mevalonate pathway-associated genes. This threshold of 81 is the top 2.5th percentile of the number of mevalonate pathway genes positively correlated with each gene in the microarray dataset. These 53 genes will be simply termed 'NFY-correlated genes' below.

A literature search found no reported direct physical association between NFY and any of the 53 gene products. However, according to DAVID, many of these genes were related to DNA metabolic processes, DNA repair, or mRNA metabolism (Table IV). To account for the observed associations between NFY and NFY-correlated genes, known protein interactions were sought using Genes2Networks (25). Fig. 3 shows the overall network, formed by NFY genes, NFY-correlated genes, and intermediate genes which connect these two gene sets. Extracts from the network, subnets 1 and 2, are shown in Figs. 4 and 5, respectively. Subnet 1 has 15 NFY-correlated 
Table V. List of genes in subnets 1 and 2 that were positively associated with the NFY-correlated genes.

Symbol Name

Genes in subnet 1

ASPM ASP (abnormal spindle) homolog, microcephaly associated (Drosophila)

CPSF6 Cleavage and polyadenylation specific factor 6 , $68 \mathrm{kDa}$

FBXO5 F-box protein 5

HAT1 Histone acetyltransferase 1

MCM2 MCM2 minichromosome maintenance deficient 2, mitotin (S. cerevisiae)

MDC1 Mediator of DNA damage checkpoint 1

MSH2 MutS homolog 2, colon cancer, nonpolyposis type 1 (E. coli)

MSH6 MutS homolog 6 (E.coli)

NUP153 Nucleoporin $153 \mathrm{kDa}$

ORC2L Origin recognition complex, subunit 2-like (yeast)

RFC3 Replication factor c (activator 1) 3, $38 \mathrm{kDa}$

SS18 Synovial sarcoma translocation, chromosome 18

TDG Thymine-DNA glycosylase

TRIM37 Tripartite motif-containing 37

UNG Uracil-DNA glycosylase

Genes in subnet 2

CBX1 Chromobox homolog 1 (hp1 $\beta$ homolog Drosophila)

DEK Dek oncogene (DNA binding)

LSM2 LSM2 homolog, U6 small nuclear RNA associated (S. cerevisiae)

NASP Nuclear autoantigenic sperm protein (histone-binding)

PAIP1 Poly(a) binding protein interacting protein 1

RIT1 Ras-like without caax 1

SNRPE Small nuclear ribonucleoprotein polypeptide e

TUBB Tubulin, $\beta$

UPF2 UPF2 regulator of nonsense transcripts homolog (yeast)

genes showing relatively close associations with NFY genes in the interaction network (Table V). Six such genes are involved in DNA repair and five are associated with either the cell cycle (ASPM, FBXO5), DNA metabolic processes (ORC2L, HAT1), or both (MCM3). In this subnetwork, several intermediate or 'hub' genes were closely connected to the NFY-correlated genes. Namely, PCNA and BRCA1 were connected to four of the NFY-correlated genes, and each of MCM10, PLK1, MCM2 and RPA2 to three. In addition to these hub genes, CHEK2, CDK2, MCM7, CDC6, EP300 and ORC4L were connected to two of the NFY-correlated genes as well as to two hub genes. Of these genes, PCNA, MCM2, CDK2 and MCM7 showed significantly negative correlations with EGFR (Pearson coefficients, $-0.446,-0.399,-0.381$ and -0.401 , respectively), whereas PLK1, MCM2 and CDK2 displayed significantly negative correlations with MET (Pearson coefficients, -0.373, - 0.486 and -0.495 , respectively). Moreover, the mean Pearson coef- ficients of all hub genes were -0.252 for EGFR and -0.240 for MET, both of which were significantly lower than the means for all genes in the dataset (-0.0089 for EGFR and -0.0313 for MET; $\mathrm{P}=1.678 \mathrm{E}-4$ and 0.0029 by t-tests, respectively), demonstrating negative associations between hub genes and growth signals. Subnet 2 includes nine of the NFY-associated genes that were only distantly connected with NFY genes in the protein-protein interaction network. Five of these genes were related to RNA metabolic processes (PAIP1, SNRPE, DEK, UPF and LSM2) and two genes encoded proteins with histonebinding properties (NASP and CBX1). In this subnetwork, LSM1 showed high connectivity, displaying two edges with the NFY-correlated genes, and three with other intermediate genes. LSM1 is highly expressed in lung cancer and mesothelioma, and LSM1 inhibition retards tumor growth (26). Four other LMS genes were present in the subnet but there was no evidence of association with lung cancer.

\section{Discussion}

In the present study, gene expression correlation patterns predicted that mevalonate metabolism and fatty acid synthesis processes were negatively associated with expression of EGFR and MET, but positively associated with cell division. Promoter analysis suggested that the NFY transcription factor may be involved in the regulation of genes involved in mevalonate metabolism, and the processes positively associated with them. Finally, gene expression correlation patterns and protein-protein interaction data indicate that the transcriptional regulation by NFY may be mediated by its interactions with other regulators of DNA metabolic processes and cell cycle genes.

The negative correlations between growth factor signaling and lipid metabolic pathways reported here seem to indicate an inhibitory effect of cholesterol on EGFR pathways in lung adenocarcinoma. Polyunsaturated fatty acids, such as oleic acid, are also known to inhibit the EGFR pathway, although the effects depend both on particular combinations of fatty acids and the cell type (27-29). In lung adenocarcinoma, the mevalonate pathway synthesizes more non-sterol and fewer sterol products than seen in fibroblasts (30). This can result in a higher degree of prenylation of small GTP-binding proteins, and reduced levels of plasma membrane cholesterol, possibly leading to enhanced EGFR activity. Mevalonate metabolites can also influence the expression of metabolic genes through the intermediacy of the liver $\mathrm{X}$ receptor (LXR). For example, LXR can activate FDPS synthesis (31), but LXR is inhibited by geranylgeraniol (32), which is produced from isopentenylPP and farnesyl-PP. Indeed, expression of NR1H3 (LXR- $\alpha$ ) showed a significant correlation with FDPS and EBP synthesis in lung cancer samples but not in normal lung samples (data not shown), suggesting a cancer-specific regulation of mevalonate pathway genes by LXR- $\alpha$.

The positive correlations seen between the lipid metabolic pathway and cell division-related processes appear to be consistent with previous experimental evidence. Pravastatin is known to inhibit DNA synthesis, whereas addition of geranylgeranylpyrophosphate restores such synthesis and promotes the G1/S transition (33). However, inhibition of farnesyl-protein transferase induces p21 expression and G1 blockade in a p53-dependent manner, suggesting that regulation of the cell 
cycle by mevalonate metabolites occurs at both the transcriptional and translational levels. In lung carcinoma cell lines, farnesyl transferase inhibitors block farnesylation of centromeric proteins and inhibit the association of such proteins with microtubules (34). In retinoblastoma gene-deficient thyroid tumors, FDPS is overexpressed, leading to increased isoprenylation and activation of N-Ras and induction of the DNA damage response (35). These experimental findings seem to suggest that mevalonate metabolites can directly regulate the expression of genes related to cell division as well. Unsaturated fatty acids are also known to increase cell proliferation (36) (37), although the mechanism of such action is not clear. One possibility is that increased activity of intracellular signaling cascades, such as those mediated by intracellular calcium (38) or AKT (39), may enhance the response of cells to mitogenic signals. However, unsaturated fatty acids are substrates for lipid peroxidation and may cause DNA damage in lung cancer cells (40-42). This may lead, in turn, to apparent (thus not real) correlated expression of unsaturated fatty acid metabolism genes and DNA damage response genes.

Transcription factor binding sequence analysis suggested that NFY may have a considerable influence on associations of lipid metabolism genes. NFY is a ubiquitous transcriptional factor which recognizes promoter CCAAT boxes (43). NFY is known to be involved in transcriptional regulation of a wide range of genes, but the regulatory roles of NFY in lipogenesis, the cell cycle, DNA repair, and DNA synthesis are of particular interest in the present context. In lipogenic gene regulation, NFY often functions with SREBPs and SP1 (44), and recent genome-wide scanning of SREBP1, SP1 and NFY occupancy showed that NFY shares about 20 and $40 \%$ of target genes with SREBP1 and SP1, respectively, in HepG2 cells (45). In the lung adenocarcinoma dataset, some mevalonate pathway genes displayed significant correlation with SREBP1 and SREBP2, but not SP1 (data not shown), suggesting possible coordinated regulation of such genes by NFY and SREBPs in cancer cells.

The regulation of cell cycle and DNA metabolism genes by NFY is also well documented. Expression of a dominantnegative NFY subunit significantly decreased the number of cells entering the $\mathrm{S}$-phase and delayed the progress of this phase, resulting in retarded cell growth (46). NFY seems be involved in induction of S-phase-specific transcription, such as that resulting in synthesis of ribonucleotide reductase R2 (47), histone H3 (48), and cyclin B1 (49). NFY also mediates genotoxic stress-induced gene expression in a 553 -independent manner (50), and suppresses gene expression in the presence of active p53 (51), suggesting a functional dependency on co-regulators. Therefore, it was important to define proteins interacting with NFY in the lung cancer cells of the present study. Combined analysis of gene expression correlation and protein-protein interaction identified several 'hub' genes which displayed high connectivity with NFY-correlated genes and other hub genes. Importantly, many of the hub genes have been associated with lung cancer. These include BRCA1 (52,53), PCNA (54,55), PLK1 (56,57), MCM2 (58), CHEK2 (59,60), CDK2 (61) and MCM7 (62), suggesting that the network discovered here is likely to be involved in progression of lung cancer. As some such genes were also sensitive to inhibition of the mevalonate pathway [BRCA1 (63), PCNA (64), MCM2 (65), CDK2 (66) and MCM7 (67)], hub genes may also be involved in the antitumor effects of pathway inhibitors in lung cancer. These hub genes do not have direct links to NFY-correlated genes and, although functional association with NFY has been experimentally shown for BRCA1 (68), CDK2 $(49,69)$ and EP300 (70), other hub genes likely interact with NFY through intermediate genes, the expression of which was found to be correlated with that of NFY.

Finally, the results presented in this article have several important clinical implications for the treatment of lung adenocarcinoma. First, the data support the importance of lipid metabolic pathway inhibition in adenocarcinoma patients, particularly in those insensitive to anti-EGFR therapy or patients who have developed resistance to such therapy. The effects of chemotherapy may be enhanced by downregulating genes related to cell division. Some of the hub genes identified in this article are already known as lung cancer markers, but exploration of the activity of combinations of such genes should better indicate the parts of the network that are active or inactive in cancer cells, thus possibly increasing therapeutic predictive power. Finally, drugs targeting NFY may be useful to improve the efficacy of other chemotherapeutic agents, by blocking multiple pathways related to lung carcinogenesis. The roles played by NFY in a variety of cancers have been highlighted in recent reports $(71,72)$, and I believe that a new therapeutic strategy based on inhibition of NFY warrants further research and development.

\section{Acknowledgements}

This study was funded by AstraZeneca, UK.

\section{References}

1. Stinchcombe TE and Socinski MA: Current treatments for advanced stage non-small cell lung cancer. Proc Am Thorac Soc 6: 233-241, 2009.

2. Mitsudomi T and Yatabe Y: Mutations of the epidermal growth factor receptor gene and related genes as determinants of epidermal growth factor receptor tyrosine kinase inhibitors sensitivity in lung cancer. Cancer Sci 98: 1817-1824, 2007.

3. Bean J, Brennan C, Shih, JY, Riely, G, Viale A, Wang L, et al: MET amplification occurs with or without T790M mutations in EGFR mutant lung tumors with acquired resistance to gefitinib or erlotinib. Proc Natl Acad Sci USA 104: 20932-20937, 2007.

4. Yano S, Wang W, Li Q, Matsumoto K, Sakurama H, Nakamura T, et al: Hepatocyte growth factor induces gefitinib resistance of lung adenocarcinoma with epidermal growth factor receptoractivating mutations. Cancer Res 68: 9479-9487, 2008.

5. Farwell WR, Scranton RE, Lawler EV, Lew RA, Brophy MT, Fiore LD, et al: The association between statins and cancer incidence in a veterans population. J Natl Cancer Inst 100: 134-139, 2008.

6. Khurana V, Bejjanki HR, Caldito G and Owens MW: Statins reduce the risk of lung cancer in humans: a large case-control study of us veterans. Chest 131: 1282-1288, 2007.

7. Taylor ML, Wells BJ and Smolak MJ: Statins and cancer: a metaanalysis of case-control studies. Eur J Cancer Prev 17: 259-268, 2008.

8. Haukka J, Sankila R, Klaukka T, Lonnqvist J, Niskanen L, Tanskanen A, et al: Incidence of cancer and statin usage-record linkage study. Int J Cancer 126: 279-284, 2010.

9. Mantha AJ, McFee KE, Niknejad N, Goss G, Lorimer IA and Dimitroulakos J: Epidermal growth factor receptor-targeted therapy potentiates lovastatin-induced apoptosis in head and neck squamous cell carcinoma cells. J Cancer Res Clin Oncol 129: 631-641, 2003.

10. Mantha AJ, Hanson JE, Goss G, Lagarde AE, Lorimer IA and Dimitroulakos J: Targeting the mevalonate pathway inhibits the function of the epidermal growth factor receptor. Clin Cancer Res 11: 2398-2407, 2005. 
11. Kusama T, Mukai M, Iwasaki T, Tatsuta M, Matsumoto Y, Akedo $\mathrm{H}$, et al: Inhibition of epidermal growth factor-induced RhoA translocation and invasion of human pancreatic cancer cells by 3-hydroxy-3-methylglutaryl-coenzyme a reductase inhibitors Cancer Res 61: 4885-4891, 2001.

12. Park IH, Kim JY, Jung JI and Han JY: Lovastatin overcomes gefitinib resistance in human non-small cell lung cancer cells with K-Ras mutations. Invest New Drugs 28: 791-799, 2010.

13. Buhaescu I and Izzedine H: Mevalonate pathway: a review of clinical and therapeutical implications. Clin Biochem 40: 575-584, 2007.

14. Pike LJ and Casey L: Cholesterol levels modulate EGF receptormediated signaling by altering receptor function and trafficking. Biochemistry 41: 10315-10322, 2002.

15. Ringerike T, Blystad FD, Levy FO, Madshus IH and Stang E: Cholesterol is important in control of EGF receptor kinase activity but EGF receptors are not concentrated in caveolae. J Cell Sci 115: 1331-1340, 2002

16. Parmigiani G, Garrett-Mayer E, Anbazhagan R and Gabrielson E: A cross-study comparison of gene expression studies for the molecular classification of lung cancer. Clin Cancer Res 10 2922-2927, 2004.

17. Wigle DA, Jurisica I, Radulovich N, Pintilie M, Rossant J, Liu N, et al: Molecular profiling of non-small cell lung cancer and correlation with disease-free survival. Cancer Res 62: 3005-3008, 2002.

18. Rhodes DR, Yu J, Shanker, K, Deshpande N, Varambally R, Ghosh D, et al: Large-scale meta-analysis of cancer microarray data identifies common transcriptional profiles of neoplastic transformation and progression. Proc Natl Acad Sci USA 101: 9309-9314, 2004.

19. Landi MT, Dracheva T, Rotunno M, Figueroa JD, Liu H, Dasgupta A, et al: Gene expression signature of cigarette smoking and its role in lung adenocarcinoma development and survival. PLoS One 3: e1651, 2008.

20. Barrett T, Troup DB, Wilhite SE, Ledoux P, Rudnev D, Evangelista C, et al: NCBI GEO: archive for high-throughput functional genomic data. Nucleic Acids Res 37: D885-D890, 2009

21. Dennis G, Sherman BT, Hosack DA, Yang J, Gao W, Lane HC, et al: DAVID: Database for annotation, visualization, and integrated discovery. Genome Biol 4: P3, 2003

22. Huang DW, Sherman BT and Lempicki RA: Systematic and integrative analysis of large gene lists using DAVID bioinformatics resources. Nat Protoc 4: 44-57, 2009.

23. Rhee SY, Wood V, Dolinski K and Draghici S: Use and misuse of the gene ontology annotations. Nat Rev Genet 9: 509-515, 2008

24. Benjamini Y, Drai D, Elmer G, Kafkafi N and Golani I Controlling the false discovery rate in behavior genetics research Behav Brain Res 125: 279-284, 2001.

25. Berger SI, Posner JM and Ma'ayan A: Genes2networks: connecting lists of gene symbols using mammalian protein interactions databases. BMC Bioinformatics 8: 372, 2007.

26. Watson PM, Miller SW, Fraig M, Cole DJ, Watson DK and Boylan AM: CaSm (LSm-1) overexpression in lung cancer and mesothelioma is required for transformed phenotypes. Am J Respir Cell Mol Biol 38: 671-678, 2008.

27. Casabiell X,Zugaza JL,Pombo CM,Pandiella A and Casanueva FF: Oleic acid blocks epidermal growth factor-activated early intracellular signals without altering the ensuing mitogenic response. Exp Cell Res 205: 365-373, 1993.

28. McKenzie KE, Bandyopadhyay GK, Imagawa W, Sun K and Nandi S: Omega-3 and omega- 6 fatty acids and PGE2 stimulate the growth of normal but not tumor mouse mammary epithelial cells: evidence for alterations in the signaling pathways in tumor cells. Prostaglandins Leukot Essent Fatty Acids 51: 437-443, 1994.

29. Mollerup S and Haugen A: Differential effect of polyunsaturated fatty acids on cell proliferation during human epithelial in vitro carcinogenesis: involvement of epidermal growth factor receptor tyrosine kinase. Br J Cancer 74: 613-618, 1996.

30. Bennis F, Favre G, Gaillard FL and Soula G: Importance of mevalonate-derived products in the control of HMG-CoA reductase activity and growth of human lung adenocarcinoma cell line A549. Int J Cancer 55: 640-645, 1993.

31. Fukuchi J, Song C, Ko AL and Liao S: Transcriptional regulation of farnesyl pyrophosphate synthase by liver X receptors. Steroids 68: 685-691, 2003

32. Forman BM, Ruan B, Chen J, Schroepfer GJ and Evans RM: The orphan nuclear receptor LXRalpha is positively and negatively regulated by distinct products of mevalonate metabolism. Proc Natl Acad Sci USA 94: 10588-10593, 1997.
33. Terano T, Shiina T, Noguchi Y, Tanaka T, Tatsuno I, Saito Y, et al: Geranylgeranylpyrophosphate plays a key role for the G1 to $\mathrm{S}$ transition in vascular smooth muscle cells. J Atheroscler Thromb 5: 1-6, 1998.

34. Ashar HR, James L, Gray K, Carr D, Black S, Armstrong L, et al: Farnesyl transferase inhibitors block the farnesylation of CENP-E and CENP-F and alter the association of CENP-E with the microtubules. J Biol Chem 275: 30451-30457, 2000.

35. Shamma A, Takegami Y, Miki T, Kitajima S, Noda M, Obara T, et al: $\mathrm{Rb}$ regulates DNA damage response and cellular senescence through E2F-dependent suppression of N-ras isoprenylation. Cancer Cell 15: 255-269, 2009.

36. Kasayama S, Koga M, Kouhara H, Sumitani S, Wada K, Kishimoto T, et al: Unsaturated fatty acids are required for continuous proliferation of transformed androgen-dependent cells by fibroblast growth factor family proteins. Cancer Res 54 : 6441-6445, 1994.

37. Renard CB, Askari B, Suzuki LA, Kramer F and Bornfeldt KE Oleate, not ligands of the receptor for advanced glycation endproducts, promotes proliferation of human arterial smooth muscle cells. Diabetologia 46: 1676-1687, 2003.

38. Graber MN, Alfonso A and Gill DL: Recovery of $\mathrm{Ca}^{2+}$ pools and growth in $\mathrm{Ca}^{2+}$ pool-depleted cells is mediated by specific epoxyeicosatrienoic acids derived from arachidonic acid. J Biol Chem 272: 29546-29553, 1997.

39. Yun MR, Lee JY, Park HS, Heo HJ, Park JY, Bae SS, et al: Oleic acid enhances vascular smooth muscle cell proliferation via phosphatidylinositol 3-kinase/AKT signaling pathway. Pharmacol Res 54: 97-102, 2006

40. Niki E: Lipid peroxidation: physiological levels and dual biological effects. Free Radic Biol Med 47: 469-484, 2009.

41. Trombetta A, Maggiora M, Martinasso G, Cotogni P, Canuto RA and Muzio G: Arachidonic and docosahexaenoic acids reduce the growth of A549 human lung-tumor cells increasing lipid peroxidation and PPARs. Chem Biol Interact 165: 239-250, 2007.

42. Maehle L, Lystad E, Eilertsen E, Einarsdottr E, Hstmark AT and Haugen A: Growth of human lung adenocarcinoma in nude mice is influenced by various types of dietary fat and vitamin $\mathrm{E}$. Anticancer Res 19: 1649-1655, 1999.

43. Matuoka $\mathrm{K}$ and Chen KY: Transcriptional regulation of cellular ageing by the CCAAT box-binding factor CBF/NF-Y. Ageing Res Rev 1: 639-651, 2002.

44. Clarke SD: Polyunsaturated fatty acid regulation of gene transcription: a molecular mechanism to improve the metabolic syndrome. J Nutr 131: 1129-1132, 2001.

45. Reed BD, Charos AE, Szekely AM, Weissman SM and Snyder M: Genome-wide occupancy of SREBP1 and its partners NFY and SP1 reveals novel functional roles and combinatorial regulation of distinct classes of genes. PLoS Genet 4: e1000133, 2008.

46. Hu Q and Maity SN: Stable expression of a dominant negative mutant of CCAAT binding factor/NF-Y in mouse fibroblast cells resulting in retardation of cell growth and inhibition of transcription of various cellular genes. J Biol Chem 275: 4435-4444, 2000.

47. Chabes AL, Bjrklund S and Thelander L: S phase-specific transcription of the mouse ribonucleotide reductase $\mathrm{R} 2$ gene requires both a proximal repressive E2F-binding site and an upstream promoter activating region. J Biol Chem 279: 10796-10807, 2004.

48. Koessler H, Kahle J, Bode C, Doenecke D and Albig W: Human replication-dependent histone $\mathrm{H} 3$ genes are activated by a tandemly arranged pair of two CCAAT boxes. Biochem J 384: 317-326, 2004

49. Katula KS, Wright KL, Paul H, Surman DR, Nuckolls FJ, Smith JW, et al: Cyclin-dependent kinase activation and S-phase induction of the cyclin B1 gene are linked through the CCAAT elements. Cell Growth Differ 8: 811-820, 1997.

50. Jin S, Fan F, Fan W, Zhao H, Tong T, Blanck P, et al: Transcription factors OCT-1 and NF-YA regulate the p53-independent induction of the GADD45 following DNA damage. Oncogene 20: 2683-2690, 2001.

51. Manni I, Mazzaro G, Gurtner A, Mantovani R, Haugwitz U, Krause K, et al: NF-Y mediates the transcriptional inhibition of the cyclin B1, cyclin B2, and cdc25C promoters upon induced G2 arrest. J Biol Chem 276: 5570-5576, 2001.

52. Kim HT, Lee JE, Shin ES, Yoo YK, Cho JH, Yun MH, et al: Effect of BRCA1 haplotype on survival of non-small-cell lung cancer patients treated with platinum-based chemotherapy. J Clin Oncol 26: 5972-5979, 2008 
53. Boukovinas I, Papadaki C, Mendez P, Taron M, Mavroudis D, Koutsopoulos A, et al: Tumor BRCA1, RRM1 and RRM2 mRNA expression levels and clinical response to first-line gemcitabine plus docetaxel in non-small-cell lung cancer patients. PLoS One 3: e3695, 2008.

54. Volm M and Koomgi R: Relevance of proliferative and proapoptotic factors in non-small-cell lung cancer for patient survival. $\mathrm{Br}$ J Cancer 82: 1747-1754, 2000.

55. Oyama T, Osaki T, Nose N, Ichiki Y, Inoue M, Imoto H, et al: Evaluations of p53 immunoreactivity, nucleolar organizer regions, and proliferating cell nuclear antigen in non-small cell lung carcinoma. Anticancer Res 20: 505-510, 2000.

56. Zhou Q, Su Y and Bai M: Effect of antisense RNA targeting polo-like kinase 1 on cell growth in A549 lung cancer cells. J Huazhong Univ Sci Technolog Med Sci 28: 22-26, 2008.

57. Spnkuch-Schmitt B, Wolf G, Solbach C, Loibl S, Knecht R, Stegmller M, et al: Downregulation of human polo-like kinase activity by antisense oligonucleotides induces growth inhibition in cancer cells. Oncogene 21: 3162-3171, 2002.

58. Tan DF, Huberman JA, Hyland A, Loewen GM, Brooks JS Beck AF, et al: MCM2-a promising marker for premalignant lesions of the lung: a cohort study. BMC Cancer 1: 6, 2001.

59. Thompson D, Seal S, Schutte M, McGuffog L, Barfoot R, Renwick A, et al: A multicenter study of cancer incidence in CHEK2 1100delC mutation carriers. Cancer Epidemiol Biomarkers Prev 15: 2542-2545, 2006.

60. Cybulski C, Masojc B, Oszutowska D, Jaworowska E, Grodzki T, Waloszczyk P, et al: Constitutional CHEK2 mutations are associated with a decreased risk of lung and laryngeal cancers. Carcinogenesis 29: 762-765, 2008.

61. Volm M, Koomgi R and Rittgen W: Clinical implications of cyclins, cyclin-dependent kinases, RB and E2F1 in squamouscell lung carcinoma. Int J Cancer 79: 294-299, 1998.

62. Fujioka S, Shomori K, Nishihara K, Yamaga K, Nosaka K, Araki K, et al: Expression of minichromosome maintenance 7 (MCM7) in small lung adenocarcinomas (pT1): prognostic implication. Lung Cancer 65: 223-229, 2009.

63. Neville-Webbe HL, Evans CA, Coleman RE and Holen I: Mechanisms of the synergistic interaction between the bisphosphonate zoledronic acid and the chemotherapy agent paclitaxel in breast cancer cells in vitro. Tumour Biol 27: 92-103, 2006.
64. Gunning WT, Kramer PM, Lubet RA, Steele VE, End DW, Wouters W and Pereira MA: Chemoprevention of benzo(a) pyrene-induced lung tumors in mice by the farnesyltransferase inhibitor R115777. Clin Cancer Res 9: 1927-1930, 2003.

65. Morgan C, Lewis PD, Jones RM, Bertelli G, Thomas, GA and Leonard RC: The in vitro anti-tumour activity of zoledronic acid and docetaxel at clinically achievable concentrations in prostate cancer. Acta Oncol 46: 669-677, 2007.

66. Doisneau-Sixou SF, Cestac P, Faye JC, Favre G and Sutherland RL: Additive effects of tamoxifen and the farnesyl transferase inhibitor FTI-277 on inhibition of MCM-7 breast cancer cellcycle progression. Int J Cancer 106: 789-798, 2003.

67. Bruemmer D, Yin F, Liu J, Kiyono T, Fleck E, Herle AV, et al: Atorvastatin inhibits expression of minichromosome maintenance proteins in vascular smooth muscle cells. Eur J Pharmacol 462: $15-23,2003$

68. Fan W, Jin S, Tong T, Zhao H, Fan F, Antinore MJ, et al: BRCA1 regulates GADD45 through its interactions with the OCT-1 and CAAT motifs. J Biol Chem 277: 8061-8067, 2002.

69. Chae HD, Yun J, Bang YJ and Shin DY: Cdk2-dependent phosphorylation of the NF-Y transcription factor is essential for the expression of the cell cycle-regulatory genes and cell cycle $\mathrm{G} 1 / \mathrm{S}$ and G2/M transitions. Oncogene 23: 4084-4088, 2004.

70. Salsi V, Caretti G, Wasner M, Reinhard W, Haugwitz U, Engeland $\mathrm{K}$, et al: Interactions between P300 and multiple NF-Y trimers govern cyclin b2 promoter function. J Biol Chem 278: 6642-6650, 2003.

71. Goodarzi H, Elemento O and Tavazoie S: Revealing global regulatory perturbations across human cancers. Mol Cell 36: 900-911, 2009.

72. Yamanaka K, Mizuarai S, Eguchi T, Itadani H, Hirai H and Kotani H: Expression levels of NF-Y target genes changed by CDKN1B correlate with clinical prognosis in multiple cancers. Genomics 94: 219-227, 2009. 